\title{
Predicción de alturas geoidales geométricas por redes neuronales artificiales
}

\author{
José Carrión* \\ Alfonso Tierra** \\ Daniel del Cogliano ${ }^{* * *}$
}

Recibido el 18 de diciembre de 2015; aceptado el 14 de noviembre de 2016

\begin{abstract}
Level heights of the national vertical networks (NVR), is the base for the establishment of the fundamental vertical reference datum of each country. When these heights are not associated to observations of terrestrial gravity field (purely geometric characteristics), they lose usefulness in solving problems related to regional and global physical phenomena generated due to the dynamic of the Earth System. In addition, different Local Vertical Datums (LVD) does not materialize the same reference, because the determination of mean sea level (MSL) in each LVD generates discrepancies in relation to a Global Vertical Reference System (GVRS). Geometrical heights from satellite-based positioning (GNSS), could be used for the determination of physical heights whether the relationship between the reference ellipsoid (reference surface for the determination of geometrical heights) and the reference surface based on terrestrial gravity field exists. One approximation to that relation is obtained from the difference between the ellipsoidal and level heights $(h$ $H n$ ). The present study shows that the Artificial Neural Networks (ANN) are a suitable alternative for the prediction of geometrical heights based on this approximation. The multilayer ANN training was performed from the basic information come from records of the Ecuadorian NVR (NVRE) associated with GPS observa-
\end{abstract}

* Programa de Pos-graduação em Ciências Geodésicas, Setor de Ciências da Terra, Universidad Federal de Paraná, Curitiba, Brasil.

** Carrera de Ingeniería Geográfica y del Medio Ambiente, Universidad de las Fuerzas Armadas (ESPE), Sangolquí, Ecuador.

*** Facultad de Ciencias Astronómicas y Geofísicas, Universidad Nacional de La Plata, La Plata, Argentina. 
tions (GPS/Levelling). The results show that the prediction of heights has a maximum error of $7 \mathrm{~cm}$.

Key words: Physical Heights, Artificial Neural Networks, Geoidal Height, GPS/Levelling.

\section{Resumo}

As alturas niveladas pertencentes às Redes de Nivelamento Fundamental (RNF), são a base para o estabelecimento da referência vertical fundamental de cada país. Quando estas alturas não estão associadas a observações do campo de gravidade terrestre, tendo portanto características puramente geométricas, perdem utilidade na solução de problemas relacionados com fenômenos de carácter regional y global vinculados com a dinâmica do Sistema Terra. Adicionalmente, diferentes Data Verticais Locais (DVL) não materializam a mesma referência, devido a que o Nível Médio do Mar determinado em cada DVL apresenta discrepâncias em relação a um Sistema de Referência Vertical Global (SRVG). Alturas geométricas oriundas do posicionamento por satélite (GNSS), poderiam ser usadas para a determinação de alturas físicas se fosse conhecida a relação entre o elipsoide de nível (superfície de referência para as alturas geométricas) e uma superfície de referência baseada no campo da gravidade terrestre. Uma aproximação a esta relação é obtida pela diferença entre as alturas elipsoidais e niveladas $(h-H n)$. Os resultados obtidos no presente trabalho, mostram que as Redes Neurais Artificiais (RNA) são um método útil na predição da relação geométrica $h-H n$. O treinamento de uma RNA multicamada foi realizada em base à informação proveniente dos registros de RNF de Equador (RNFE) associados a observações GPS (GPS/nivelamento). Os resultados mostram que a predição de $h-H n$ pode ser realizada registrando erros máximos de $7 \mathrm{~cm}$.

Palavras chave: Alturas Físicas, Rede Neural Artificial, Altura Geoidal, GPS/nivelamento.

\section{Resumen}

Alturas niveladas pertenecientes a las Redes de Nivelación Fundamental (RNF), sirven de base para el establecimiento de la referencia vertical fundamental de cada país. Cuando estas alturas no están asociadas a observaciones del campo de gravedad terrestre, teniendo por tanto características puramente geométricas, pierden utilidad en la solución de problemas relacionados con fenómenos de carácter regional y global vinculados con la dinámica del Sistema Tierra. Adicionalmente, diferentes Data Verticales Locales (DVL) no materializan la misma referencia, debido a que el NMM determinado en cada DVL presenta discrepancias en relación a un Sistema de Referencia Vertical Global (SRVG). Alturas geométricas provenientes de posicionamiento satelital (GNSS), podrían ser usadas para la determinación de 
alturas físicas si fuese conocida la relación entre el elipsoide de nivel (superficie de referencia para las alturas geométricas) y una superficie de referencia basada en el campo de la gravedad terrestre. Una aproximación a dicha relación es obtenida de la diferencia entre las alturas elipsoidales y niveladas $(h-H n)$. Los resultados obtenidos en el presente trabajo muestran que las Redes Neuronales Artificiales (RNA) son un método promisorio en la predicción de la relación geométrica $h-H n$. El entrenamiento de una RNA multicapa ha sido realizado, la información de base proviene de registros de la RNF de Ecuador (RNFE) asociados a observaciones GPS (GPS/nivelación), los resultados muestran que la predicción de $h-H n$ puede ser realizada registrando errores máximos de $7 \mathrm{~cm}$.

Palabras clave: Alturas Físicas, Red Neuronal Artificial, Altura Geoidal, GPS/nivelación.

\section{El Sistema de Alturas en Ecuador}

En el Ecuador Continental la materialización de la red de nivelación se ha realizado tomando como datum de referencia, al mareógrafo ubicado en La Libertad, Provincia de Santa Elena. Los puntos de nivelación están situados principalmente sobre la red vial con la finalidad de facilitar el acceso a los mismos. La corrección por efecto de la gravedad sobre las alturas niveladas de la red altimétrica, aún no se ha ejecutado, pese a que en cada uno de los puntos pertenecientes a la red de nivelación se cuenta con información gravimétrica enlazada a la red gravimétrica fundamental, esto determina que en Ecuador no se disponga de alturas referidas a una superficie equipotencial sino únicamente alturas obtenidas tomando como referencia el nivel medio del mar (alturas niveladas) que presentan un error kilométrico estimado de $4 \mathrm{~mm}$ y una tolerancia de cierre de $4 \mathrm{~mm} \sqrt{k}$ (Leiva, 2005).

Complementariamente se han desarrollado campañas GPS sobre los puntos de la RNFE, en la primera de ellas las mediciones fueron hechas con equipos de baja precisión (navegadores) con el objetivo de localizar los registros gravimétricos. Posteriormente se han realizado nuevas mediciones con receptores de doble frecuencia, alcanzando precisiones con valores entre 2 y $5 \mathrm{~cm}$, en la determinación de las coordenadas geocéntricas. Las coordenadas se encuentran referidas al Marco de Referencia SIRGAS (ITRF 94), época de referencia 1995.4 (Leiva, 2005).

El ajuste de la red altimétrica aún no está realizado, en tales circunstancias existirá un error intrínseco en los datos que será evidenciado en la evaluación de los resultados. En el año de 1948 se instala en Ecuador la estación mareográfica de La Libertad, para dar inicio a la determinación del dátum vertical con asesoramiento de IAGS (Interamerican Geodetic Survey) del Ecuador. En 1960 se calcula el nivel medio del mar usando datos extraídos de mareogramas correspondientes al período 1950 -1959. Una vez obtenido el nivel medio del mar, el Instituto Geográfico Mili- 
tar (IGM), mediante nivelación geométrica transportó las cotas a la gran mayoría de ciudades del país. En 1970, la estación mareográfica de La Libertad pasa a depender del INOCAR (Instituto Oceanográfico de la Armada), organismo que desde entonces ha realizado semestralmente las inspecciones mareográficas, cambio y nivelación de la regla de mareas a hitos establecidos en tierra (Paredes, 1986).

Las alturas niveladas con que cuenta la red altimétrica en Ecuador, presentan discrepancias con respecto a alturas determinadas mediante redes de nivelación de otros países, explicándose este hecho por estar referida la red altimétrica nacional a un datum local. En Ecuador aún no se encuentra establecido un sistema de alturas que esté referido a una superficie equipotencial, en un futuro el sistema de referencia vertical en el país debería estar orientado al cálculo de números geopotenciales, según lo recomienda la International Association of Geodesy (IAG), con lo cual se conseguiría el establecimiento de un sistema de alturas físico (International Height Reference System - IHRS), (IAG, 2015).

\section{Método geométrico para el cálculo del geoide}

El método geométrico para la obtención del geoide se basa en el cálculo de alturas geoidales $(\eta)$ en función de alturas elipsoidales y alturas niveladas. El conocimiento del comportamiento del geoide geométrico en sitios puntuales por medio de este método permite extender el cálculo del mismo en lugares que no se dispone de observaciones de alturas elipsoidales y alturas niveladas; se incursiona de esta forma en el campo de la estimación de variables. Este método se origina de la relación entre alturas ortométricas y elipsoidales (Ecuación 1).

$$
h \approx H+\eta
$$

La relación presentada en la Ecuación 1 no es lineal, ya que las alturas elipsoidales $(h)$ son medidas sobre la normal y las alturas ortométricas $(H)$ sobre la vertical. Las alturas ortométricas involucran el conocimiento del campo de gravedad local, ya que esta condición no se cumple para el área de trabajo contemplada en el presente estudio, la altura $H$ presente en la Ec. 1 es aproximada por alturas provenientes de nivelación geométrica $(\mathrm{Hn})$.

Las alturas geoidales tienen una connotación fundamentalmente física, debido a que están referidas a una superficie equipotencial del campo de gravedad terrestre, por lo tanto, es necesario señalar que en el presente trabajo, las llamadas alturas geoidales geométricas no cumplen con esta definición, ya que se obtienen de la comparación de dos medidas de carácter geométrico. 


\section{Alturas niveladas}

Las alturas niveladas $(\mathrm{Hn})$ son aquellas que se obtienen de las redes de nivelación de un país y tienen como referencia un datum altimétrico relacionado con el Nivel Medio del Mar (NMM) determinado por un mareógrafo. El NMM permite considerar el conjunto de variaciones estacionales que pueda presentar el mismo. Una vez que se determina el origen del sistema de referencia vertical, se realiza nivelación hacia el interior del continente, con la finalidad de establecer puntos de control sobre la superficie topográfica (ST) para los cuales se fija un valor de altura a través del cálculo de desniveles $(\Delta H n)$ (Figura 1$)$. Posteriormente estos puntos nivelados son usados como referencia para establecer valores de altura en nuevos sitios. Para la nivelación de las redes altimétricas nacionales se usa por lo general el método geométrico siendo este el de mejor desempeño en comparación con la nivelación trigonométrica en términos de precisiones alcanzadas.

La suma algebraica de desniveles medidos en un circuito de nivelación y asumiendo la no existencia de errores en la medición, no es igual a cero, esto se debe al no paralelismo de las superficies de nivel involucradas en la nivelación, en general los desniveles determinados generan resultados que dependen del trayecto recorrido durante la determinación de desniveles (Hofmann y Moritz, 2005).

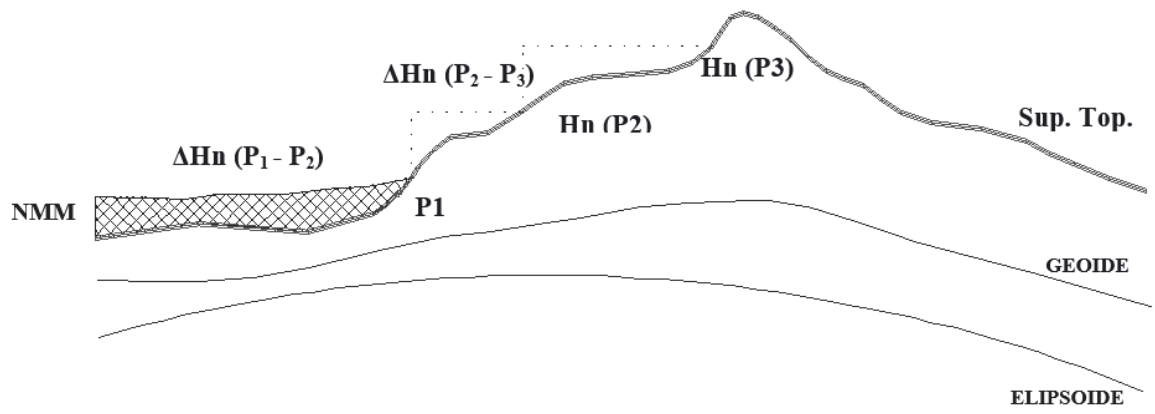

Figura 1. Alturas Niveladas.

\section{Alturas elipsoidales}

Las alturas elipsoidales son medidas sobre la normal al elipsoide desde su superficie, este tipo de alturas tienen un carácter geométrico y no pueden ser usadas en trabajos para los cuales sea requisito la determinación de alturas considerando aspectos físicos en la determinación de los desniveles.

La altura geoidal $(\eta)$ es la distancia que existe entre un elipsoide de referencia (elipsoide de nivel) y el geoide como superficie de referencia para las alturas físicas. Según la definición dada por Gauss-Listing el geoide es una superficie equipo- 
tencial del campo de gravedad terrestre que mejor se ajusta al nivel medio de los mares de acuerdo con la teoría de los mínimos cuadrados (Heck, 2004).

En la Figura 2 se puede apreciar como en base al conocimiento de las alturas niveladas y las alturas elipsoidales, es posible llegar a calcular los valores de altura geoidal.

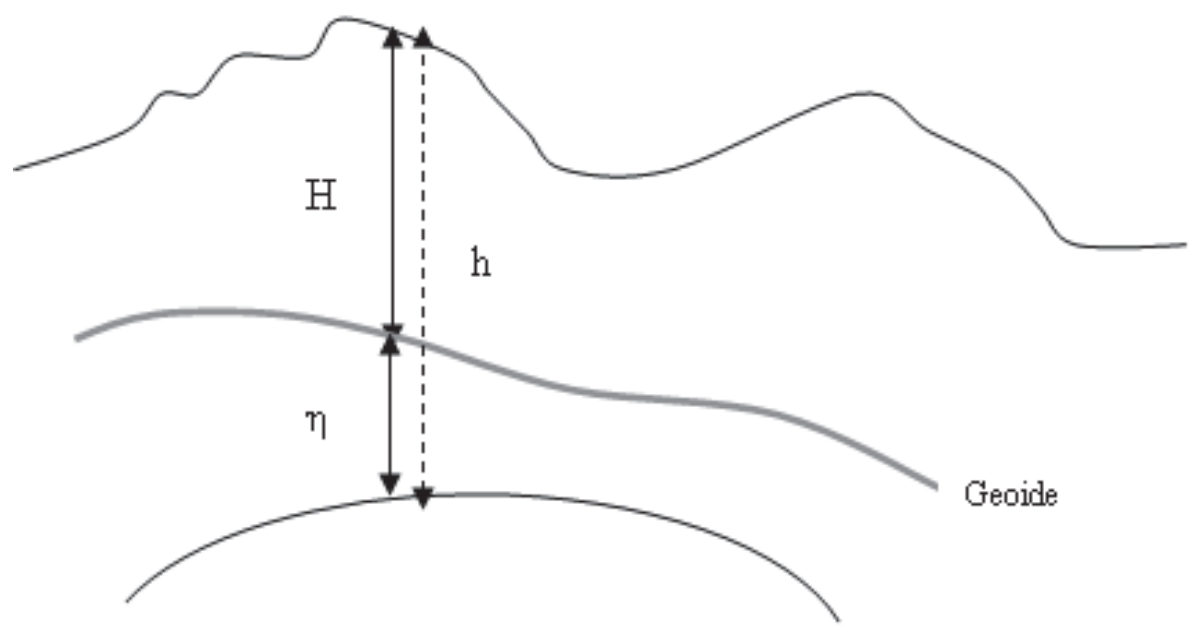

Figura 2. Superficies de Referencia.

\section{Redes Neuronales Artificiales (RNAs)}

El funcionamiento del cerebro animal, desde siempre ha constituido un enigma y ha sido objeto de innumerables estudios e investigaciones; la capacidad del cerebro en tareas de resolución de problemas involucra una serie de funciones internas que todavía no son comprendidas en su totalidad.

La aparición de los sistemas informáticos permitió que muchas de las tareas con las que se enfrentaba a diario el ser humano sean realizadas con mayor agilidad y eficiencia, sin embargo este tipo de sistemas presentan una limitación, la resolución de problemas requiere un tratamiento algorítmico, cuando el problema a solucionar no se acopla a estas características, es necesario que se de otro tipo de tratamiento al procesamiento de la información que participa en el asunto al que se quiera dar solución (Isasi y Galván, 2004).

La Inteligencia Artificial es la disciplina que estudia los mecanismos mediante los cuales una máquina puede emular el funcionamiento del cerebro humano, con el fin de dar solución a problemas que los sistemas informáticos clásicos no pueden hacer. Constituye un intento por adaptar las cualidades cognoscitivas del ser humano al campo informático, de tal forma que se pueda conjugar la gran capacidad 
de resolución de problemas del cerebro con la agilidad de los procesos informáticos. Se puede afirmar que aquellos problemas que no admiten una resolución algorítmica, nos conducen a una solución que se basa en la capacidad de memorizar y asociar hechos, es decir que use como insumo fundamental la experiencia.

Una RNA, es un conjunto de unidades procesadoras que reciben el nombre de neuronas y se encuentran interconectadas para permitir el intercambio y procesamiento de información (Basogain, 2008). En el ámbito de la Geodesia y áreas afines, las RNAs han ganado espacio principalmente como un método de estimación de variables. Específicamente en el caso de Ecuador, el trabajo pionero en el empleo de técnicas de inteligencia artificial para fue generado para la tesis doctoral titulada: "Metodologia para a geração da malha de anomalias gravimétricas para obtenção de geoide gravimétrico local a partir de dados esparsos" (Tierra y De Freitas, 2003), en dicho trabajo se verifica un óptimo desempeño de una RNA multicapa entrenada para predecir anomalías gravimétricas sobre una grilla, como requisito para el posterior cálculo de un geoide local mediante el método gravimétrico.

\section{Neurona artificial}

Las neuronas artificiales, células o autómatas son capaces de cambiar de estado dependiendo de las entradas que tengan y de las conexiones sinápticas asociadas, este estado se conoce como nivel de activación y significa que ante nuevas entradas la red podrá presentar estados de activación diferentes. Las neuronas cuentan con una función que les hace posible cambiar de estado de activación a partir de las señales que reciben, esta función se conoce como función de activación o función de transición de estado, el nivel de activación de una célula depende de las entradas que reciba y de los valores de las conexiones sinápticas, pero no depende de estados de activación anteriores (Isasi y Galván, 2004).

Para determinar el nivel de activación de una neurona $(y)$, es necesario en primer lugar calcular la entrada total a la célula para posteriormente evaluar este valor por medio de la función de transferencia $(f)$, la entrada total a la neurona $(\Sigma)$ es equivalente a la suma ponderada de las entradas por sus respectivos pesos sinápti$\cos (\omega)$ (Figura 3).

La entrada total a la célula puede definirse por la Ecuación 2:

$$
\Sigma=x_{1} * \omega_{1}+x_{2} * \omega_{2}+x_{3} * \omega_{3}+\ldots \ldots . .+x_{n} \omega_{n}
$$

Y en forma vectorial:

$$
\Sigma=X^{T} * \omega
$$


En donde:

$X^{T}=$ vector de entradas a la neurona

$\omega=$ vector de pesos sinápticos

Luego de aplicar a $\Sigma$ una función de trasferencia $f$, se calcula la salida de la neurona según la Ecuación 4.

$$
y=f(\Sigma)
$$

La estructura y componentes básicos de una neurona artificial se observan en la Figura 3.

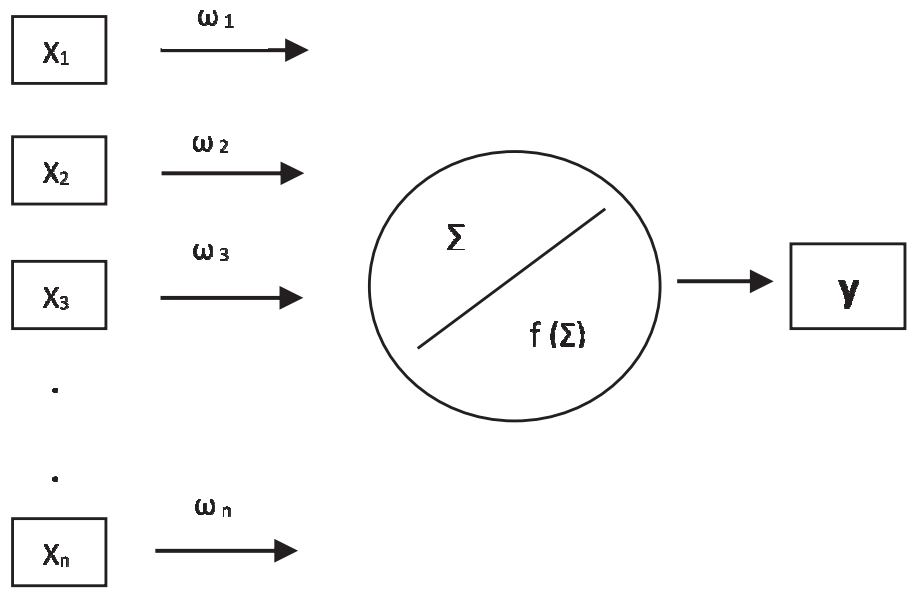

Figura 3. Modelo de neurona artificial.

La función de activación o función de transferencia $(f)$ produce la salida $(y)$ de la neurona al evaluar el valor de $\Sigma$, existen distintas funciones de transferencia y dependiendo de cuál se utilice se define el modelo de neurona artificial que se requiera, en general puede ser cualquier función y su elección dependerá del tipo de fenómeno involucrado en el aprendizaje de la neurona (Haykin, 2001).

\section{Entrenamiento de una RNA}

El aprendizaje de la red sucede durante el entrenamiento, es decir la red aprende a reconocer patrones relacionándolos con sus respectivas salidas, los pesos sinápticos son ajustados en varias iteraciones mientras se ingresan las entradas del conjunto de 
entrenamiento y se comparan sus salidas con las que genera la red. Un algoritmo de aprendizaje permite ajustar los pesos sinápticos en función de las diferencias existentes entre las salidas reales o targets y las salidas calculadas por la red, existe una variedad de algoritmos válidos para realizar el entrenamiento y su elección depende del tipo de problema que la red debe aprender a solucionar.

Los algoritmos de aprendizaje se basan en la resolución del problema de mínimos cuadrados no lineales, se trata de encontrar los pesos sinápticos que minimicen una función de error. Los algoritmos calculan variaciones para los pesos sinápticos mediante un proceso iterativo que minimiza la función de error (Haykin, 2001).

\section{Area de estudio y datos usados}

El área en la cual se desarrolla el trabajo (Figura 4), corresponde a una región delimitada en función de la distribución espacial de los datos, la misma incluye provincias de la región Sierra y Costa del Ecuador Continental. El área tiene una extensión aproximada de $24,344.48 \mathrm{~km}^{2}$ y un rango de alturas niveladas de $3 \mathrm{~m}$ a 5,245 m sobre el NMM.

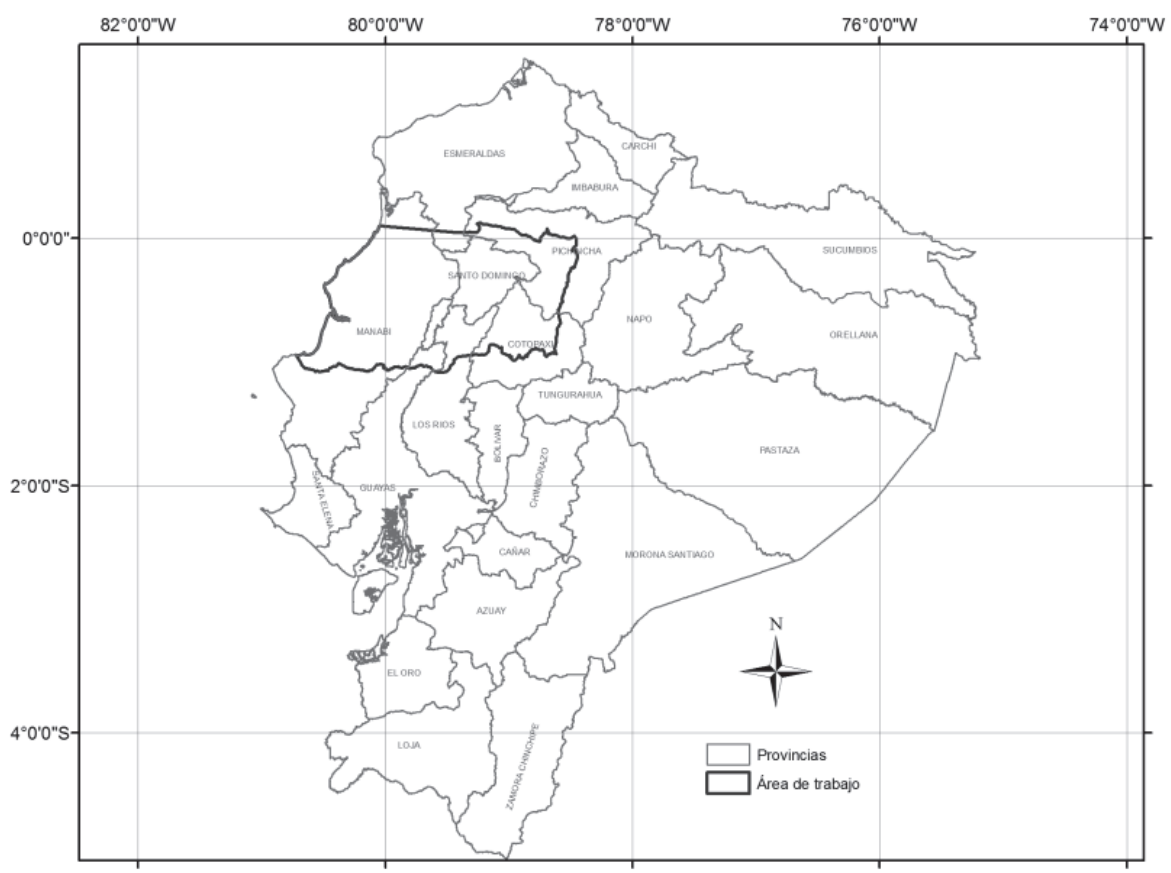

Figura 4. Localización del área de trabajo. 
La información requerida para realizar el entrenamiento de la RNA son puntos de la RNFE ocupados con posicionamiento GPS (GPS/nivelación). Los datos de altura nivelada y altura elipsoidal permiten calcular los valores de altura geoidal geométrica en cada punto. Esta información complementada con las coordenadas geodésicas horizontales, conforman el conjunto de entrenamiento de la RNA; de esta forma los parámetros de posición constituyen las entradas de la red y los valores de altura geoidal sus salidas correspondientes.

Previo a la realización del trabajo se recopilaron 57 puntos GPS/nivelación de precisión preexistentes y relevados por el Instituto Geográfico Militar de Ecuador (IGM). Para conseguir una mejor distribución de los datos en el área de estudio, se ejecutó una nueva campaña GPS para el presente estudio, en colaboración con personal de la Escuela Politécnica del Ejército de Ecuador (ESPE). Solo aquellos registros GPS que cumplían con precisiones asociadas a las alturas elipsoidales menores de $10 \mathrm{~cm}$ y que disponían de alturas niveladas por el método geométrico, fueron considerados en el análisis. Un total de 72 puntos fueron empleados para el desarrollo de la metodología propuesta (Figura 5).

El objetivo del entrenamiento de la red fue alcanzar un nivel de generalización que hiciera posible estimar alturas geoidales en puntos con coordenadas geodésicas conocidas.

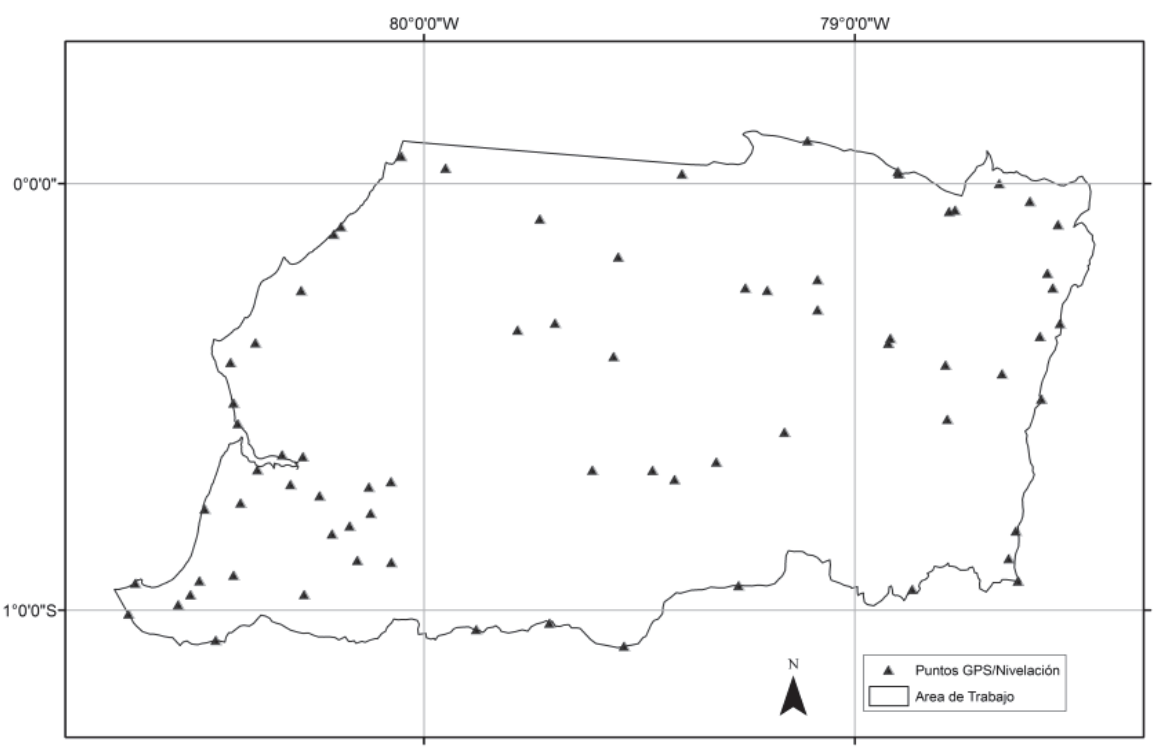

Figura 5. Distribución de los puntos GPS/nivelación. 
Con la finalidad de homogeneizar los registros GPS, las coordenadas geodésicas de los datos que provienen de las campañas realizadas previamente por el IGM y de los nuevos puntos obtenidos en la nueva campaña, fueron transformadas al sistema de referencia ITRF05 y época de referencia 2011.1.

Para realizar el entrenamiento de la RNA, fue necesario definir tres subconjuntos del total de datos disponibles, un grupo de datos para el entrenamiento propiamente dicho (58 registros), otro conjunto de validación (siete registros) y un tercer conjunto de test (siete registros). La selección de los subconjuntos se realizó considerando que estos deben ser representativos, es decir que los ejemplos empleados en la etapa de aprendizaje de la RNA deben ser diversos y equiparables en proporción, es decir los registros deberán representar un amplio rango de patrones que para que abarquen la variabilidad del fenómeno estudiado. Adicionalmente y en relación directa con el grado de representatividad de los ejemplos, los subconjuntos deben ser significativos, es decir contar con un número apropiado de ejemplos (Basogain, 2008).

Los datos del conjunto de entrenamiento son empleados para realizar el ajuste de los pesos sinápticos mediante el algoritmo de aprendizaje y en base a la comparación de sus salidas deseadas y las salidas que genera la red, las salidas generadas por la RNA para el conjunto de validación fueron un indicador de la capacidad de generalización de la RNA. Los datos del conjunto de entrenamiento ingresaron iterativamente a la red durante el entrenamiento mientras la modificación en los pesos sinápticos repercutió en una reducción de los errores que genera la misma para los datos del conjunto de validación; es por este motivo que se considera que los datos del conjunto de validación participan en el aprendizaje de la RNA y no son un indicador absoluto de la capacidad de generalización de la misma.

Por lo tanto se hace necesario contar con un conjunto de datos que no influencien de ninguna forma en el proceso de aprendizaje de la RNA, de tal forma que los errores que se generen para estos datos, brinden una estimado sin sesgos de la capacidad de generalización de la red, este es el subconjunto de test.

\section{Configuración y aprendizaje de la $R \mathrm{NA}$}

La configuración definitiva de la RNA entrenada se determinó mediante un procedimiento de prueba y error, es decir, se experimentó con diferentes configuraciones de red en busca de aquella que proporcionaba resultados acordes a las expectativas del método. Distintas variantes de configuraciones de red fueron evaluadas en función de su desempeño, el número de capas ocultas o intermedias, el número de neuronas en cada una de estas capas y las funciones de transferencia presentes en las neuronas artificiales, constituyen las principales variantes en cada una de las configuraciones de RNA probadas. 
En función de las características particulares del problema tratado, la capa de entrada de la RNA está constituida por las variables: latitud, longitud y altura elipsoidal y la capa de salida por una neurona que corresponde a la variable que debe ser estimada (altura geoidal). El proceso de prueba y error determinó que la configuración óptima de la RNA consta de tres capas interconectadas completamente, una capa oculta con 24 neuronas, para la capa oculta se definió una función de transferencia tangente sigmoidal y para la capa de salida una función de transferencia lineal. El tratamiento no lineal del problema se consigue mediante la configuración de dos capas de neuronas y una capa de entradas (RNA multicapa), con una capa oculta cuyas salidas son generadas por una función de transferencia tangente sigmoidal (Figura 6). El algoritmo de aprendizaje (minimización de función de error mediante la modificación de los pesos sinápticos) usado fue el de Levenberg Marquardt, la función de error a minimizar por medio del algoritmo de entrenamiento es el error medio cuadrático (Mean Square Error - MSE) (Carrión et al., 2013).

Con el objetivo de optimizar el proceso de aprendizaje, las entradas y salidas del conjunto de entrenamiento fueron normalizadas (media cero y desviación estándar 1), debido a que el entrenamiento de la RNA es realizado en función de estos valores normalizados, es importante destacar que la predicción de alturas geoidales se debe realizar también en base a entradas normalizadas, y de la misma forma los valores de salida generados por la RNA deben ser sometidos a una desnormalización para obtener sus valores reales.

\section{Resultados}

El entrenamiento óptimo de la RNA se consiguió en 16 iteraciones, lográndose el mejor desempeño de la red en la iteración número 10; en la Figura 7 es posible apreciar como los errores (MSE calculado en base a salidas normalizadas) en los subconjuntos de entrenamiento, validación y test disminuyen paulatinamente conforme avanza el proceso iterativo. Se considera que la RNA ha alcanzado un aprendizaje adecuado, cuando su capacidad de generalización se evidencia en errores aceptables (esperados) cuando se evalúa el desempeño de la red con los elementos del conjunto de test (Carrión et al., 2013). El conjunto de test (línea roja, Figura 7) es útil para tener un indicador no sesgado del desempeño de la red.

Después de establecer el conjunto óptimo de pesos sinápticos, la RNA está en capacidad de predecir valores de altura geoidal en posiciones que no fueron consideradas dentro del conjunto de entrenamiento. Los estadísticos de los errores generados por la RNA entrenada para los diferentes subconjuntos considerados son lo presentados en la Tabla 1, donde se puede observar que los errores extremos (desnormalizados) en la predicción de alturas geoidales en ningún caso superan los $8 \mathrm{~cm}$. Los valores de error cuadrático medio (MSE), en los cuales se basa el aprendizaje de la RNA se ubican en 1e-03 para el caso de los conjuntos de entrenamiento y validación, y en 2e-03 para los registros de test. 


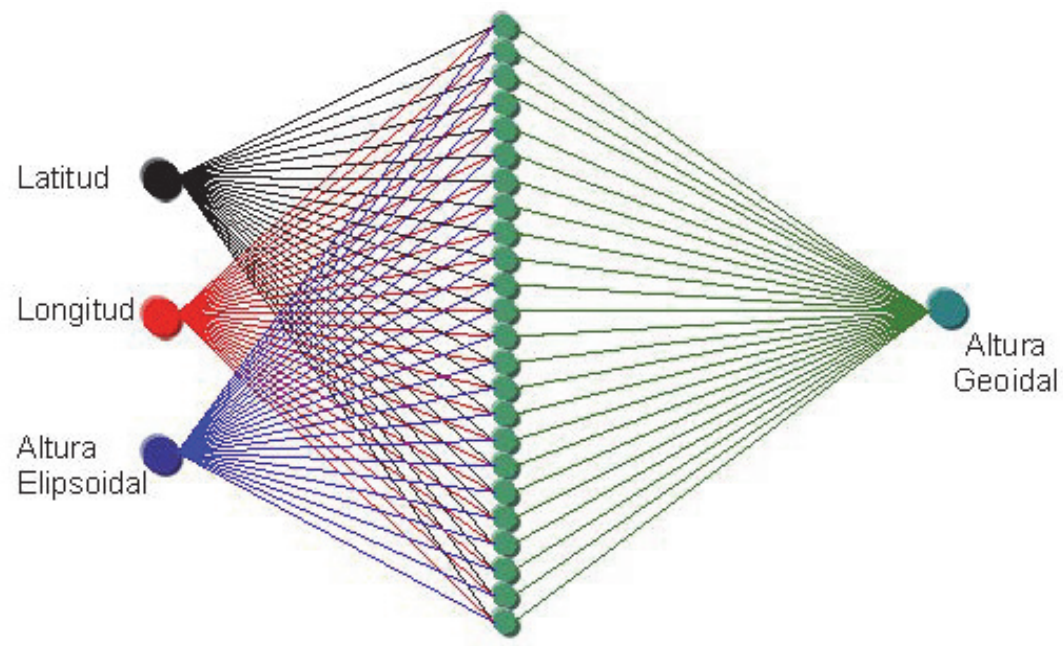

Figura 6. RNA entrenada.

Performance (plotperform)

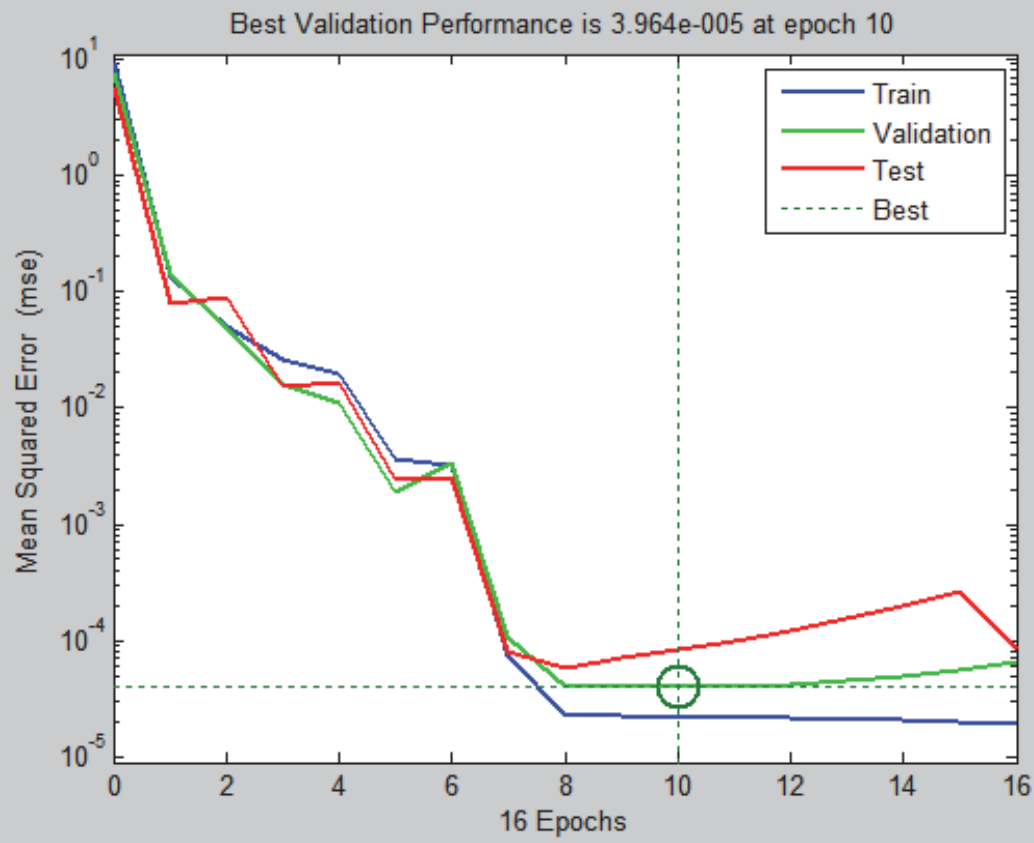

Figura 7. Minimización de errores en los subconjuntos de entrenamiento, validación y test. 
Tabla 1

Estadísticos para (N_GPS/niv - N_RNA)

\begin{tabular}{lccc}
\hline & $\begin{array}{c}\text { Entrenamiento } \\
\text { sin normalizar }\end{array}$ & $\begin{array}{c}\text { Validación sin } \\
\text { normalizar }\end{array}$ & $\begin{array}{c}\text { Test sin } \\
\text { normalizar }\end{array}$ \\
\hline Máximo (m) & 0.063 & 0.024 & 0.072 \\
Mínimo (m) & -0.075 & -0.061 & -0.062 \\
Media (m) & 0.000 & -0.007 & -0.007 \\
Desviación & 0.025 & 0.034 & 0.051 \\
Estándar & 0.001 & 0.001 & 0.002 \\
MSE & & & \\
\hline
\end{tabular}

El desempeño de la RNA, fue contrastado con la predicción de alturas geoidales para los registros del conjunto de test, mediante el modelo EGM2008 con grado de desarrollo en armónicos esféricos ( $\mathrm{n}=2160$ ) (Pavlis et al., 2008), que representa el estado del arte en cuanto a Modelos Globales de Geopotencial (MGGs). En la Tabla 2 , se presentan los estadísticos calculados para las diferencias entre las alturas geoidales correspondientes al modelo (N_EGM2008) y aquellas derivadas de GPS/niv.

Tabla 2

Estadísticos para N_GPS/niv - N_EGM2008

\begin{tabular}{cc}
\hline Errores para los registros de test evaluados con EGM2008 $(\mathrm{m})$ \\
\hline Máximo & 1.310 \\
Mínimo & 0.172 \\
Media & 0.608 \\
Desviación Estándar & 0.355 \\
\hline
\end{tabular}

Los estadísticos para las diferencias $N_{-} G P S / n i v$ - $N_{-} E G M 2008$ calculadas para los puntos de test, muestran en general un desempeño inferior que el conseguido por la RNA, las diferencias se concentran en torno de $10560 \mathrm{~cm}$, lo cual difiere significativamente con el valor medio de $5 \mathrm{~cm}$ en el caso del cálculo realizado con las salidas de la RNA entrenada.

\section{Conclusiones}

- El empleo de redes neuronales artificiales demuestra ser una herramienta apropiada en la estimación del comportamiento del geoide, al ser comparada con otros métodos cuando se dispone de datos dispersos.

- Es factible pensar en la adición de otras variables relacionadas con el fenómeno estudiado, como por ejemplo gravedad, anomalías gravimétricas, desviaciones 
de la vertical, etc., con la finalidad de incrementar la cantidad de información de que dispone la red neuronal para lograr su aprendizaje.

- Es importante que los datos disponibles para realizar el entrenamiento de la red neuronal sean representativos, es decir, es necesario que la información contenida por estos abarque la mayor cantidad de patrones que definan el rango real de valores que pueden presentar las variables intervinientes dentro del área de estudio.

- Los datos de test y validación deben ser representativos y significativos al igual que los del conjunto de entrenamiento, de tal forma que los resultados obtenidos en la evaluación del desempeño de la red sean consistentes.

- La metodología propuesta en el presente trabajo para un área determinada, es replicable en otras zonas, siempre y cuando se disponga de un conjunto de datos de entrenamiento adecuado.

- La precisión de los datos empleados para el entrenamiento de la red neuronal condiciona la calidad que se tendrá en las salidas proporcionadas por la misma luego de su entrenamiento.

- La red neuronal artificial de mejor aprendizaje, se determina en base a un proceso de prueba error, es decir se adopta como óptima a aquella red que durante el entrenamiento logra un mejor desempeño en cuanto se refiere a la minimización los errores.

- El método GPS/nivelación y RNAs muestra ser capaz de generar un modelo geoidal local con un rango de error menor a $8 \mathrm{~cm}$. El desempeño de la RNA entrenada dependerá directamente de la calidad y características del conjunto de datos disponibles (representativos y significativos).

\section{Bibliografia}

Basogain X. (2008). "Redes neuronales artificiales y sus aplicaciones [Informe]", Departamento de Ingeniería de Sistemas y Automática, Escuela Superior de Ingeniería de Bilbao, Bilbao [s.n.], p. 79.

Carrión, J.L.; Tierra, A. y Del Cogliano, D. (2013). “Generación de una malla de ondulaciones geoidales por el método GPS/nivelación y redes neuronales artificiales a partir de datos dispersos [Informe]", tesis de maestría, Facultad de Ciencias Astronóminas y Geofísicas, Universidad Nacional de La Plata, UNLP, La Plata.

Haykin S. (2001). Redes Neurais, princípios e prática, Ontario, Bookman.

Heck, B. (2004). "Problems in the Definition of Vertical Reference Frames", Hotine-Marussi Symposium on Mathematical Geodesy (IAG Symposia), ed. Sansó F.- Berlín, Springer, vol. 127.

Hofmann, B. y Moritz, H. (2005), Physical Geodesy, Graz, Springer, 2:158. 
IAG (2015). Resolution No. 1, for the definition and realization of an International Height Reference System (IHRS) [Informe], Resolution / IAG-[s.1.], p. 2.

Isasi, P. y Galván, I. (2004). Redes Neuronales Artificiales. Un enfoque práctico, Madrid, Pearson Educación S.A.

Leiva César A. (2005). "Proyecto SIRGAS Ecuador [Informe]", Instituto Geográfico Militar de Ecuador, Caracas, Reuniones Técnicas SIRGAS, p. 26.

Paredes, Nelson (1986). "Acta Oceanográfica del Pacífico-Guayaquil", Determinación del Datum Vertical de La Libertad, Ecuador, INOCAR, 1(3):205-209.

Pavlis, N. et al. (2008). "An Earth Gravitational Model to Degree 2160: EGM2008”, Informe técnico, Vienna, s.n.

Tierra, A. y De Freitas, S.R.C. (2003). "Metodologia para a geração da malha de anomalias gravimétricas para obtenção de geoide gravimétrico local a partir de dados esparsos", tesis doctoral, Departamento de Geomática, Universidade Federal do Paraná, Curitiba, Universidade Federal do Paraná. 